\title{
Preparation of Small RNA Libraries for Sequencing from Early Mouse Embryos
}

\author{
Rachel A. Keuls ${ }^{1}$, Ronald Parchem ${ }^{1}$ \\ ${ }^{1}$ Development, Disease Models \& Therapeutics Graduate Program, Department of Molecular and Cellular Biology, Department of Neuroscience, Center for \\ Cell and Gene Therapy, Stem Cells and Regenerative Medicine Center, Baylor College of Medicine
}

\section{Corresponding Author}

Ronald Parchem

Ronald.Parchem@bcm.edu

\section{Citation}

Keuls, R.A., Parchem, R. Preparation of Small RNA Libraries for Sequencing from Early Mouse Embryos. J. Vis. Exp. (164), e61086, doi:10.3791/61086 (2020).

\section{Date Published}

October 9, 2020

\section{DOI}

$10.3791 / 61086$

URL

jove.com/video/61086

\section{Abstract}

MicroRNAs (miRNAs) are important for the complex regulation of cell fate decisions and developmental timing. In vivo studies of the contribution of miRNAs during early development are technically challenging due to the limiting cell number. Moreover, many approaches require a miRNA of interest to be defined in assays such as northern blotting, microarray, and qPCR. Therefore, the expression of many miRNAs and their isoforms have not been studied during early development. Here, we demonstrate a protocol for small RNA sequencing of sorted cells from early mouse embryos to enable relatively unbiased profiling of miRNAs in early populations of neural crest cells. We overcome the challenges of low cell input and size selection during library preparation using amplification and gel-based purification. We identify embryonic age as a variable accounting for variation between replicates and stage-matched mouse embryos must be used to accurately profile miRNAs in biological replicates. Our results suggest that this method can be broadly applied to profile the expression of miRNAs from other lineages of cells. In summary, this protocol can be used to study how miRNAs regulate developmental programs in different cell lineages of the early mouse embryo.

\section{Introduction}

A central question of developmental biology is how a single undifferentiated cell can give rise to an entire organism with numerous complex cell types. During embryogenesis, the developmental potential of cells becomes progressively restricted as the organism develops. One example is the neural crest lineage, which progressively differentiates from a multipotent cell population into various terminal derivatives, such as peripheral neurons, glia, cranial bone, and cartilage. Neural crest cells are specified from the ectoderm during gastrulation and then undergo an epithelial to mesenchymal transition and migrate through the embryo to discrete locations throughout the body where they will terminally differentiate ${ }^{1}$. Decades of work have uncovered a transcriptional gene regulatory network, but far less is known 
about the mechanisms of post-transcriptional regulation that control the timing of neural crest development.

Previous work suggests that microRNAs (miRNAs) repress gene expression for proper developmental timing and cell fate decisions ${ }^{2,3,4,5,6}$. Studies of miRNAs in neural crest development have largely focused on later stages of craniofacial development. For example, miR-17 92 and miR-140 are critical for palatogenesis during craniofacial development in mouse and zebrafish, respectively ${ }^{7,8}$. The contribution of miRNAs to the earliest neural crest fate decisions of the embryo has not been thoroughly investigated. Studies of miRNAs in early fate decisions have been limited by technical challenges such as the low cell number present in early embryos.

MiRNAs have been profiled in vitro from cell lines using embryoid bodies at different stages of differentiation to model early mouse development ${ }^{9}$. The investigation of small RNAs in vivo during early mammalian development has been relatively limited. Previous methods to profile miRNAs have led to bias as a known sequence is used to analyze expression of a specific miRNA in methods such as qPCR, microarrays, and northern blots ${ }^{10}$. Next generation sequencing and ever improving molecular tools now allow for relatively unbiased analysis of miRNA expression to study their contribution to early mammalian development and cell fate decisions.

Here, we report a technique to harvest and sequence small RNAs expressed in neural crest cells from early mouse embryos spanning gastrulation (E7.5) to the beginning of organogenesis (E9.5). This technique is straightforward and combines lineage tracing, cell sorting, and gel-based size selection to prepare small RNA sequencing libraries from a minimal number of cells for next generation sequencing.
We highlight the importance for strict somite stage matching of embryos to resolve 6-hour time intervals to obtain a comprehensive view of miRNAs during the rapid changes of early development. This method can be widely applied to genetic and developmental studies and avoids the pooling of embryos. We describe a way to overcome challenges of current methods such as miRNA enrichment using gelbased purification, library quantification, and minimizing bias introduced from PCR. This method has been used to identify miRNA expression patterns over time to study how miRNAs control developmental timing in the neural crest lineage of mouse embryos.

\section{Protocol}

All research and animal care procedures were approved by the Baylor College of Medicine Institutional Animal Care and Use Committee and housed in the Association for Assessment and Accreditation of Laboratory Animal Careapproved animal facility at Baylor College of Medicine. All strains were maintained on C57BL6 background.

\section{Embryo dissection (E7.5-E9.5)}

1. Remove uterine horns from a pregnant female mouse, sterilizing the abdomen with $70 \%$ ethanol where the incision is to be made.

2. Clean off the uterus by rinsing with phosphate buffered saline (PBS). Place the uterus into a sterile plastic $10 \mathrm{~cm}$ dish.

3. Using microdissection scissors, separate each decidua containing region from one another. Peel off the uterine muscle and expose all deciduae. One by one, remove the decidua from each embryo. 
4. Remove the yolk sac from each embryo and save for genotyping.

5. Move all embryos into a new dish of fresh PBS for imaging.

6. Take an image of the entire litter. Image each embryo individually and count the number of somites of each embryo. Keep the magnification and exposure (for fluorescence) the same between experiments.

NOTE: We find that $50-200$ ms exposure is adequate for fluorescence and that $20 \mathrm{~ms}$ is adequate for bright field setting.

\section{Embryo dissociation and cell sorting}

1. For each embryo to be sorted from, do the following.

1. Decapitate just above the otic placode for embryos older than E8.0 (if only labeled cells of the cranial region are desired). For E7.5 embryos, remove extraembryonic structures (if only the embryo proper is desired).

2. Move the head in a minimal volume of PBS over to a clean well of a 48 -well plate. Add $250 \mu \mathrm{L}$ of papain (27 U/mL). Pipette up and down gently using a p200.

3. Check under the microscope and look for clumps and single cells.

4. Repeat gentle pipetting up and down until a single cell suspension is achieved (usually three rounds of pipetting up and down which should equate to between $30 \mathrm{~s}$ to $1 \mathrm{~min}$ for E7.5-E9.5).

5. Quench with $250 \mu \mathrm{L}$ of fetal bovine serum (FBS).

6. Repeat steps 2.1.1-2.1.5 for each embryo to be sorted.
2. Filter $500 \mu \mathrm{L}$ of cell suspension through a $35 \mu \mathrm{m}$ nylon mesh filter cap tube to remove clumps.

3. Take the filtrate and move to new $1.5 \mathrm{~mL}$ tube and spin at $200 \times g$ for $5 \mathrm{~min}$. Remove supernatant carefully and transfer to new tube (save until live cells are known to be in the pellet).

4. Resuspend cell pellet in $300 \mu \mathrm{L}$ of PBS containing $0.5-1 \%$ bovine serum albumin and keep on ice until sorting (minimize the time between now and the end of sorting).

1. If desired, take $10 \mu \mathrm{L}$ of cell suspension and combine with $10 \mu \mathrm{L}$ of Trypan blue (0.4\%) and count using a hemocytometer to identify approximate cell quantification and viability as Trypan blue only stains dead cells.

5. Just before sorting, filter each sample again through a filter cap tube and add DAPI stain for live cells.

6. Sort each sample into $500 \mu \mathrm{L}$ of RNA extraction lysis solution (see Table of Materials) on cell sorter with 70 $\mu \mathrm{m}$ nozzle.

7. Mix and store at $-80{ }^{\circ} \mathrm{C}$ until all samples are harvested. Proceed from this point only when all samples needed for an experiment are harvested to reduce technical variation between rounds of library preparation.

\section{RNA extraction}

NOTE: The protocol is adapted from the RNA isolation kit; see Table of Materials.

1. Add $500 \mu \mathrm{L}$ of RNA extraction lysis solution (see Table of Materials) to each sample to make the total volume $1000 \mu \mathrm{L}$.

2. Thaw each sample at room temperature. 
3. Vortex each sample for $1.5 \mathrm{~min}$, making sure that the cap is securely on each tube before starting homogenization. Incubate the homogenate at room temperature (15-25 $\left.{ }^{\circ} \mathrm{C}\right)$ for $5 \mathrm{~min}$.

4. Add $140 \mu \mathrm{L}$ of chloroform, cap tube securely and shake vigorously for $15 \mathrm{~s}$. Incubate at room temperature for 3 $\min$.

5. Centrifuge for $15 \mathrm{~min}$ at $12,000 \times g$ at $4{ }^{\circ} \mathrm{C}$. Transfer the upper aqueous phase to a new collection tube on ice. Do not transfer any interphase or any organic phase. When approaching the pink organic phase, tip the tube to side and remove small aliquots until no clear aqueous phase can be collected.

6. Measure the volume of RNA containing aqueous phase collected from each sample for the correct calculation in the next step.

7. Add 1.5 volumes (usually $525 \mu \mathrm{L}$ but may be slightly more or less) of $100 \%$ ethanol and mix thoroughly by pipetting.

8. Pipette up to $700 \mu \mathrm{L}$ sample, including any precipitate, into column in a $2 \mathrm{~mL}$ collection tube. Close the lid and centrifuge at $\geq 8,000 \times g$ for $15 \mathrm{~s}$ at room temperature. Discard the flow-through.

9. Repeat step 3.8 using the remainder of the sample.

10. Wash the column as per manufacturer instructions. Dry the column by spinning at full speed for $1 \mathrm{~min}$. Transfer the column to a new $1.5 \mathrm{~mL}$ collection tube.

11. Pipette $11 \mu \mathrm{L}$ of nuclease free water onto the center of the membrane. Let sit at room temperature for $1 \mathrm{~min}$. Spin max speed for 1 min to elute off the column.

12. Measure RNA concentration using a spectrophotometer and a parallel capillary electrophoresis instrument (Table of Materials).

\section{Library preparation}

NOTE: The protocol is adapted from small RNA library preparation kit handbook; see Table of Materials.

1. Complete the denaturation and 3 ' adapter ligation as stated in the small RNA library preparation kit handbook using $1 / 4$ dilution of 3 adapter.

2. Continue to excess 3 ' adapter removal.

3. Complete the adapter depletion as stated in the small RNA library preparation kit handbook. Be sure to use freshly prepared $80 \%$ ethanol for the bead cleanup and that all excess ethanol is completely removed just prior to the resuspension in nuclease-free water without overdrying the beads (cracking of the bead pellet is observed upon over-drying).

4. Proceed directly to excess adapter inactivation.

5. Complete the excess adapter inactivation as stated in the small RNA library preparation kit handbook assembling all reagents on ice.

6. Continue to $5^{\prime} 4 \mathrm{~N}$ adapter ligation.

7. Complete $5^{\prime}$ adapter ligation as stated in the small RNA library preparation kit handbook being sure to use a $1 / 4$ dilution of the 5' adapter and assembling all reagents on ice.

8. Proceed to reverse transcription.

9. Complete reverse transcription-first strand synthesis as stated in the small RNA library preparation kit handbook and taking care to assemble all reagents on ice and not keeping the enzyme out of the freezer for extended periods of time. 
NOTE: At this point, the protocol may be stopped, with samples stored overnight at $4{ }^{\circ} \mathrm{C}$. Alternatively continue to PCR amplification.

10. Complete PCR amplification by assembling the reagents as stated in the small RNA library preparation kit handbook and minimizing the number of PCR cycles. The number of PCR cycles should be experimentally determined for each application and the minimum number of cycles should be used. Here we used 16 cycles to successfully amplify our small RNA libraries.

11. Proceed directly to size selection.

\section{Size selection}

1. To each sample, add $5 \mu \mathrm{L}$ of $6 \mathrm{x}$ gel loading dye and pipette to mix.

2. Load $10 \mu \mathrm{L}$ of ladder to the first well of the gel, leaving the well immediately to the right empty. Load all of product from step 5.1 onto a $6 \%$ Tris/borate/ ethylenediaminetetraacetic acid - polyacrylamide gel (TBE-PAGE) and leave 1 lane in between each sample. NOTE: Keep the container that the gel came in for subsequent steps.

3. Run the gel at $150 \mathrm{~V}$ for approximately $30-40$ min in $0.5 x$ TBE running buffer (until the lower dye band is near the bottom of the gel, $0.5-1 \mathrm{~cm}$ ).

4. Make $1 \mathrm{~L}$ of staining solution while the gel is running: SYBR Gold diluted 1:10,000 in 0.5x TBE.

5. When the gel has finished running (i.e., lower dye band is near the bottom of the gel), carefully remove the gel from the glass plates, noting the orientation of the gel.

6. Place gel in the tray of its original packaging. Remove the $0.5 x$ TBE and replace with the staining solution from step
5.5. Stain gel for $15 \mathrm{~min}$. Staining time may need to be increased.

7. Place the gel on a UV transilluminator, taking care to not rip the gel and maintaining the orientation noted above (re-stain for additional time if bands of ladder cannot be clearly seen).

8. Image the gel once staining is complete on UV transilluminator with a camera.

1. If a better image is required, first use an imaging apparatus other than the UV transilluminator to capture an image of the gel before cutting out the bands on a UV transilluminator as imaging directly on the UV transilluminator causes the variable coloring of the background as shown in Figure 3A-B. Do not move the gel too many times as it is delicate and may tear.

9. Identify and remove the $\sim 150$ bp band using a clean razor blade and place into clean $1.5 \mathrm{~mL}$ tube. Do not cut out the $\sim 130 \mathrm{bp}$ band (adapter dimer product).

1. Image the gel again after removing the slices containing the library product for documentation purposes.

10. Spin the microcentrifuge tubes containing the gel slice at max speed for $30 \mathrm{~s}$ to collect the slices at the bottom of each tube.

11. Use a p200 tip for each sample to crush the slice of gel into the smallest bits possible. Eject each tip into each tube.

12. To each tube, add $300 \mu \mathrm{L}$ of elution buffer, taking care to wash gel bits from side of tube and reattach the p200 tip and wash off the tip for each sample.

13. Place the eluting samples in a shaking incubator set to 25 ${ }^{\circ} \mathrm{C}$. Incubate overnight with $1000 \mathrm{rpm}$ shaking. Remove 
tubes from incubator and spin at max speed for $10 \mathrm{~min}$ at room temperature.

14. Transfer the supernatant containing the library product to a $2 \mathrm{~mL}$ RNase free 96 well plate. Take care to not transfer any gel.

15. Add $50 \mu \mathrm{L}$ cleanup beads to each sample and mix by pipetting. Immediately, add $350 \mu \mathrm{L}$ of isopropanol and mix by pipetting. Incubate at room temperature for 10 minutes with rocking.

16. Pulse spin plate to pellet beads and then magnetize for 2 min. Carefully remove and discard the supernatant.

17. Add $950 \mu \mathrm{L}$ of $80 \%$ ethanol. Incubate for $30 \mathrm{~s}$. Remove all of the supernatant. Repeat for a total of two ethanol washes.

18. Dry the sample for $3 \mathrm{~min}$. At this step take care to remove any residual ethanol that collects at the bottom of each well (not more than once) and to not over-dry the beads (over-drying will result in cracking of the bead pellet).

19. Remove all residual liquid at the bottom of the well. Remove the plate from the magnetic stand.

20. Resuspend the pellet in $13 \mu \mathrm{L}$ of resuspension buffer. Mix by pipette until homogeneous. Incubate for $2 \mathrm{~min}$ and then magnetize for $3 \mathrm{~min}$.

21. Transfer $12 \mu \mathrm{L}$ of supernatant to a clean tube or well of clean plate for subsequent storage. Store all samples at $4{ }^{\circ} \mathrm{C}$ overnight or $-20^{\circ} \mathrm{C}$ long term.

22. Check the size and concentration of fragments present in each library using the capillary electrophoresis high sensitivity DNA assay. Confirm the concentration with more than one method.
NOTE: We have occasionally used the standard sensitivity kit to avoid overloading the capillary electrophoresis.

\section{Representative Results}

Using the procedure demonstrated here, we have harvested embryos at E7.5, E8.5, and E9.5. Extraembryonic structures were removed from all embryos and then embryos were somite staged to resolve 6-hour time intervals (Figure 1A-1B). Using principle component analysis to group samples based on similarity, we find that samples cluster by age, highlighting the variation as a result of embryonic age and the need for careful somite matching for biological replicates (Figure 1C). We profiled the pluripotent epiblast at E7.5, lineage traced premigratory and migratory neural crest cells using Wnt1-Cre at E8.5 and migratory neural crest cells using Sox10-Cre at E9.5 (Figure 1D). Here we specifically harvest the cranial neural crest by decapitating the embryo just above the otic placode. A comparison of the two Credrivers (Wnt1 and Sox10) that are frequently used to label neural crest cells confirms they mark different populations in early mouse embryos (Figure 1E). Gating strategies were used to obtain live single RFP positive cells which were sorted directly into RNA extraction lysis solution (see Table of Materials) and stored at $-80^{\circ} \mathrm{C}$ (Figure 1F). It is important to keep track of how many cells were harvested from each sample.

RNA isolation was performed using a modified version of the RNA kit protocol. Specifically, we use the mini RNA columns that are to be stored at $4{ }^{\circ} \mathrm{C}$. These columns are useful for eluting in a small volume $(11 \mu \mathrm{L})$ to obtain the highest possible concentration of RNA from samples where cell input is limiting. For this same reason, it is important to obtain all of the aqueous phase after the phenol chloroform 
extraction. Slow pipetting and tilting the tube to one side while collecting are critical to maximize yield. In this procedure, we quantify the RNA using both a spectrophotometer, to obtain information on salt/protein contamination, and a capillary electrophoresis to measure concentration (Figure 2). The spectrophotometer trace reveals that RNA was isolated with no contaminating proteins but has high salt content (Figure 2A-2B). Ideally the $260 / 280$ ratio should be $\sim 1.8$ and the $260 / 230$ ratio should be $>2.0$. The total RNA yield as measured by the spectrophotometer did not increase with age between E8.5-E9.5. This is due to the resolution of the spectrophotometer not being sensitive enough to detect changes in RNA concentration from the number of cells that we are harvesting, and we recommend using the concentration information obtained from the capillary electrophoresis (Figure 2C). The capillary electrophoresis trace can be used to estimate concentration and size of the RNA fragments. Peaks $<1000$ nucleotides are indicative of degradation. The representative trace is consistent with RNA that is not degraded. Peaks at 2000 nucleotides and 5100 nucleotides are $18 \mathrm{~s}$ and $28 \mathrm{~s}$ rRNA, respectively. The small RNA region is located at $\sim 150$ nucleotides (Figure 2D).

Small RNA sequencing libraries were prepped using the small RNA library prep kit described in the Table of Materials. Here we use just less than half of the RNA obtained from each sample ( $4 \mu \mathrm{L}$ of the $11 \mu \mathrm{L}$ elution, $\sim 80-120 \mathrm{ng}$ ) that enables RNA-sequencing libraries to be synthesized from the remaining RNA from each sample. Here we dilute the 3' and 5' small RNA adapters $1 / 4$ to lower the amount of adapter dimers present and suggest that the adapter ligation, cleanup, and reverse transcription steps are completed as much as possible in a continuous manner. We have used 16 PCR cycles to amplify the libraries and suggest that the minimum number of PCR cycles for any experiment be empirically determined. By completing excess PCR cycles, one could artificially inflate the read count of a lowly expressed miRNA.

Size selection is imperative to enrich for libraries of miRNAs and not adapter dimers, which are typically present. The library product size (150 bp) and adapter dimers (130 bp) are very similar in size. Gel extraction is used to isolate the small RNA sequencing libraries away from the adapter dimers (Figure 3). An image of a PAGE gel before excision shows that a multitude of product sizes are present in each sample (Figure 3A). It is important to leave one lane open between any two samples or a ladder that is loaded onto the gel. The migration front at the bottom of the gel is slightly curved indicating that running at a slower speed may be necessary if the $150 \mathrm{bp}$ band is difficult to distinguish for all samples. This representative image also shows an abundance of $150 \mathrm{bp}$ product from the higher concentration of positive control RNA (total RNA from the brain of a rat) that went into the library prep as compared to that which went into each embryonic sample. The negative control reveals that the reagents were free of contaminating nucleic acid species (Figure 3A). Excision of the $150 \mathrm{bp}$ band should be done with a clean razor blade and the area collected is shown in Figure 3B. Capillary electrophoresis traces before and after size selection show the dramatic improvement of the purity of the $150 \mathrm{bp}$ library product with gel purification (Figure 3C-3D). It is important to note that the traces in Figure 3C-3D have sample peaks higher than the markers, indicative of overloading. In these cases, the size of the fragments can be accurate, however the concentration may not. Quantification can be obtained by diluting the libraries into the range of the markers or using alternative methods. We find some variation across alternative methods of library quantification and recommend that multiple methods of quantification be empirically tested. Accurate quantification of concentration 
is essential when maximizing the number of samples to be sequenced at one time. Libraries will be diluted down to $1.3 \mathrm{pM}$ for sequencing and generally anywhere from 15-25 samples can be sequenced at one time on a 150 cycle sequencing kit that provides $\sim 140$ million reads. This results in about 5 million reads per sample. Generally mapping rates are between $60-80 \%$. 
A

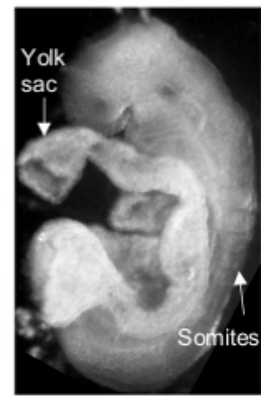

D

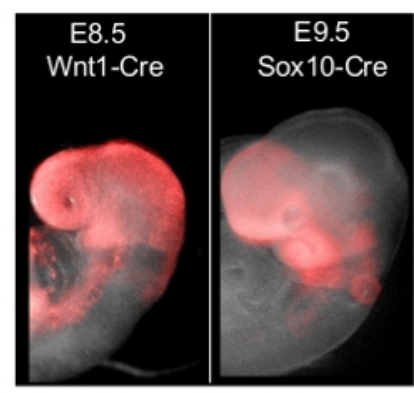

$\mathbf{F}$
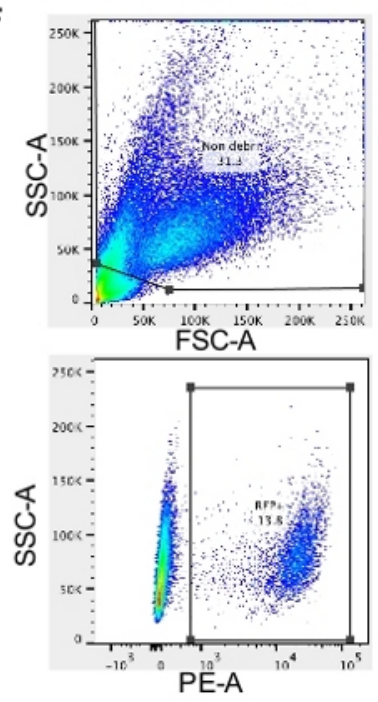

B

\begin{tabular}{|l|l|}
\hline Somites & Stage \\
\hline $0-2$ & E8.0 \\
\hline $3-4$ & E8.25 \\
\hline $5-7$ & E8.5 \\
\hline $8-12$ & E8.75 \\
\hline $13-20$ & E9.0 \\
\hline $21-24$ & E9.5 \\
\hline
\end{tabular}

E
C

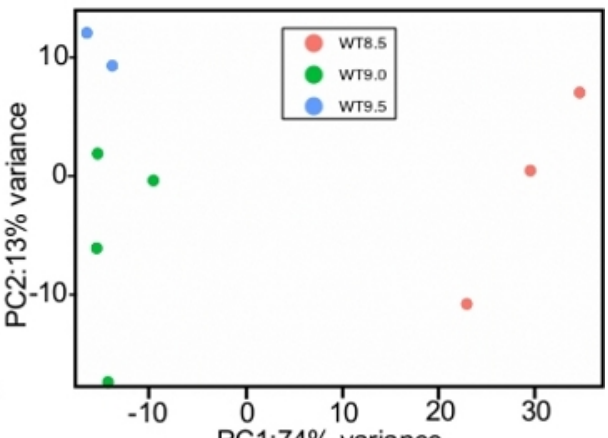

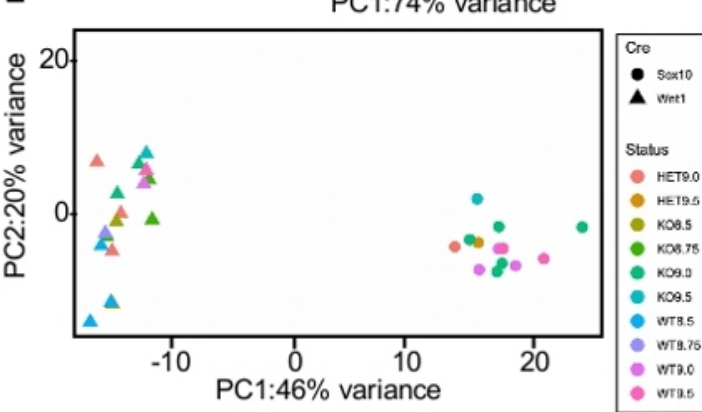
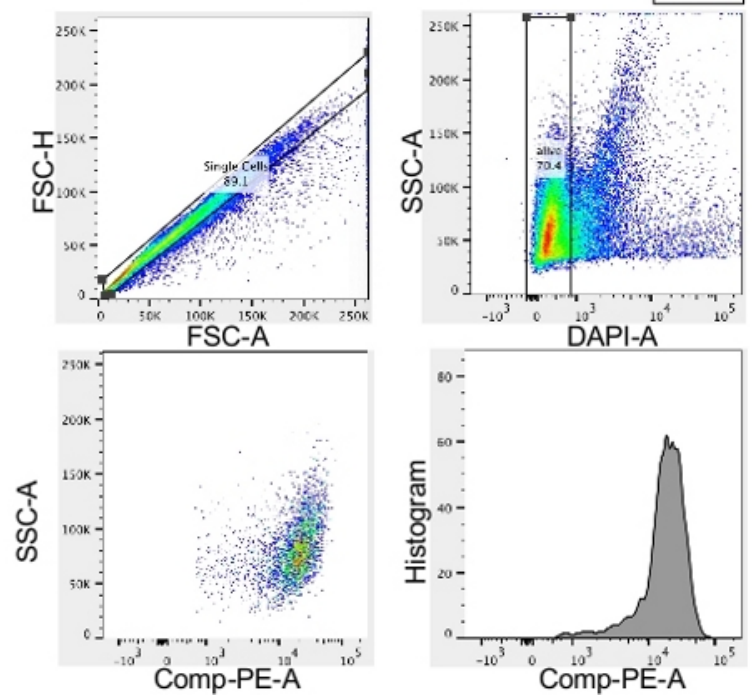

Figure 1: Harvesting cells from mouse embryos for small RNA sequencing. (A) E8.5 mouse embryo to highlight the removal of the yolk sac and somites used to determine the stage of the embryo (B) Somite staging of mouse embryos to capture the stages of neural crest development (C) Principle component analysis of libraries prepped from sorted wildtype neural crest cells showing that samples group by age (D) Schematic showing how samples were harvested using Wnt1-Cre at E8.5 to label premigratory and migratory neural crest cells and Sox10-Cre at E9.5 to label only migratory neural crest cells (E) Principle component analysis of libraries prepped from sorted wildtype neural crest cells showing samples group together by Cre-driver regardless of age (F) Gating strategy used to isolate RFP+ neural crest cells from E8.5 and E9.5 embryos using FACS sorting. Please click here to view a larger version of this figure. 
A

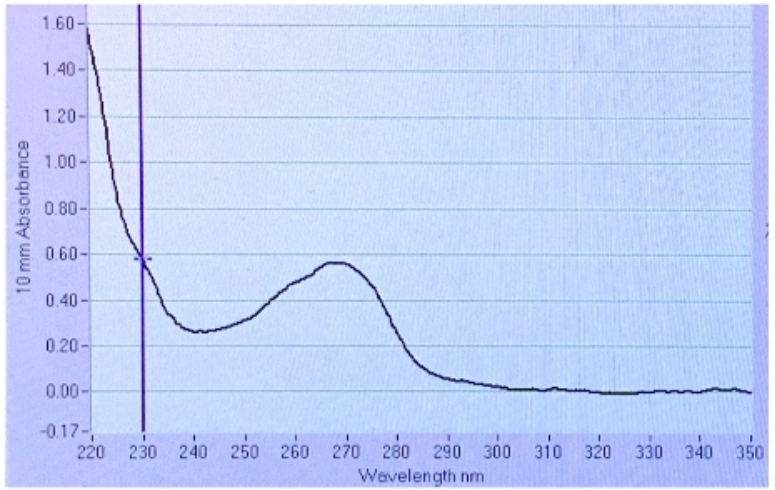

\begin{tabular}{|c|c|}
\hline Absorbance & 0.578 \\
\hline A260 10mm path & 0.480 \\
\hline A280 10mm path & 0.258 \\
\hline $260 / 280$ & 1.86 \\
\hline $260 / 230$ & 0.83 \\
\hline Concentration $(\mathrm{ng} / \mu \mathrm{L})$ & 19.2 \\
\hline
\end{tabular}

C

\begin{tabular}{|c|c|c|c|c|c|}
\hline $\begin{array}{c}\text { Cre- } \\
\text { Driver }\end{array}$ & Age & Somites & $\begin{array}{c}\text { Cell } \\
\text { Number }\end{array}$ & $\begin{array}{c}\text { RNA } \\
\text { Conc. }(\mathrm{ng} / \mu \mathrm{L})\end{array}$ & $\begin{array}{c}\text { Total RNA } \\
\text { yield }(\mathrm{ng})\end{array}$ \\
\hline Wnt1 & E8.5 & 5 & 3023 & 29.1 & 320.1 \\
\hline Wnt1 & E8.75 & 10 & 5224 & 23.3 & 256.3 \\
\hline Wnt1 & E9.5 & 21 & 10972 & 22.7 & 249.7 \\
\hline
\end{tabular}

D

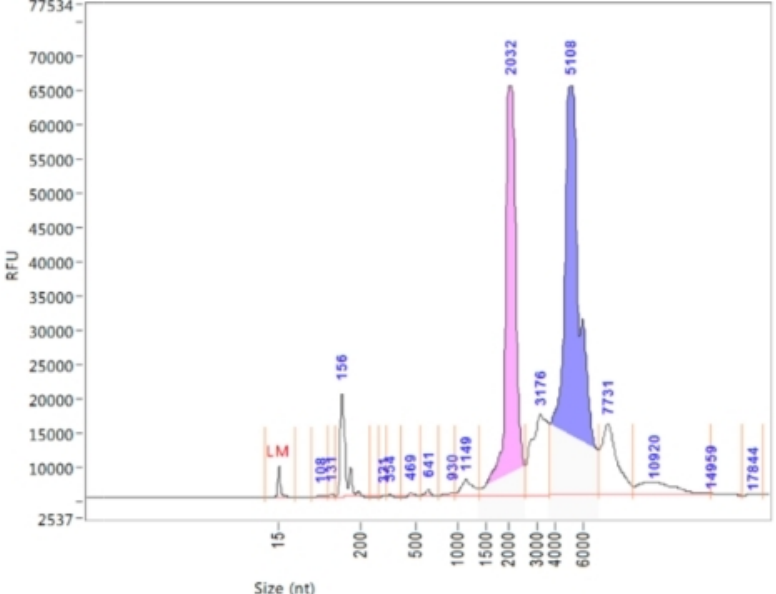

Figure 2: RNA isolation from E7.5-E9.5 mouse embryos. (A) Representative spectrophotometer trace of an RNA isolation from the youngest stage of embryo that we have applied this protocol. (B) Table showing the representative values of RNA quality obtained from the spectrophotometer. (C) Example of the RNA harvested from sorted neural crest cells from embryos of each age (D) Capillary electrophoresis trace showing good quality RNA. Please click here to view a larger version of this figure. 


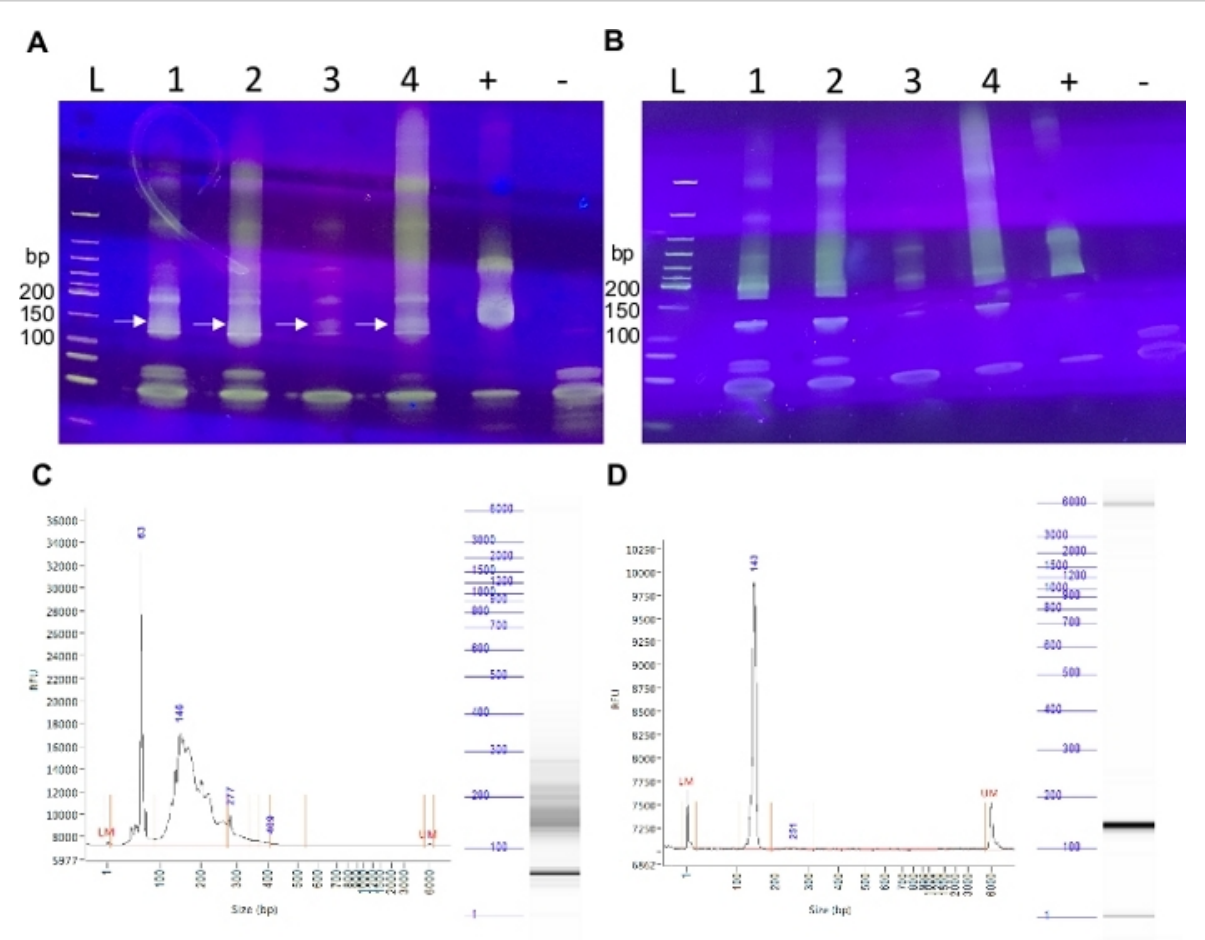

Figure 3: miRNA libraries before and after size selection. (A) TBE-PAGE gel showing representative small RNA libraries for four samples and controls before and (B) after $150 \mathrm{bp}$ band excision. The arrows represent the $150 \mathrm{bp}$ band to be excised (C) Capillary electrophoresis trace of small RNA library before and (D) after size selection. Please click here to view a larger version of this figure.

\section{Discussion}

Developmental processes can proceed rapidly, and cells are undergoing many sequential specifications such that to capture a comprehensive view of the miRNAs contributing to early fate decisions, more specific staging is needed than the widely used half-day increment. A recent study has performed RNA sequencing from Theiler stage 12 embryos which range from having 3 to 6 somites ${ }^{11}$. We find that during this period of time, the neural crest cells are specified ( 3 somites of age), delaminate (4 somites of age), and migrate (5-6 somites of age). We also find that besides the Credriver used to lineage trace cell populations, age is the largest source of variation between biological samples, and only somite matched embryos should be considered as replicates. This should also be taken into consideration when comparing transgenic embryos to wildtype controls.

Previous methods to profile miRNAs during early development have used between 10-100 ng of RNA input for small RNA sequencing library preparation and have pooled multiple embryos into one sample ${ }^{13,14}$. We demonstrate RNA isolation and library preparation from a single E7.5 embryo or from sorted neural crest cells at E8.5 and E9.5 using approximately $100 \mathrm{ng}$ of total RNA as input. When dissociating embryos for sorting, one should take care to watch the dissociation under a microscope and quench the 
reaction to observe when single cells are obtained. We find that dissociation of the cranial region of E8.5-E9.5 embryos is almost instant with gentle manual pipetting as described in the protocol. For larger tissues and increasingly older embryos, dissociation time may be longer depending on the portion of the embryo being dissociated. For E7.5-E9.5 embryos, clumps of cells are easily visible under the microscope and the dissociation should continue until no more clumps are visible. Single cells are visible in solution if you adjust the focus through the solution in your well anywhere from 5-10x. Previous methods sort cells directly into lysis buffer for RNA sequencing to prep bulk RNA from a low number of cells ${ }^{14}$. Here we sort directly into RNA extraction lysis solution so that RNA can be isolated before the start of library preparation. Use of mini columns with $11 \mu \mathrm{L}$ elution volume allowed for a high enough RNA concentration such that a single RNA prep could be split between small RNA and bulk RNA sequencing.

One current limitation of most small RNA sequencing methods is the PCR amplification of converted cDNA. Our method does not overcome this limitation, but we were able to minimize the number of PCR cycles from the 25maximum recommended down to 16 cycles. This reduction in amplification decreases artificial amplification bias introduced by PCR. Another source of bias is the ligation of adapters, where it is known that specific sequences located at the ends of adapters and miRNAs can ligate together with greater efficiency than other sequences. To avoid this, the adapters used in this protocol have 4 random bases incorporated at the end of each adapter to prevent bias in ligation reactions. Additionally, another common issue is the amount of adapter dimers that form when the RNA input is low. The library preparation kit does include steps to reduce adapter dimer formation such as adapter inactivation and bead cleanups to remove excess adapters after each ligation. We also diluted the $3^{\prime}$ and 5' $4 \mathrm{~N}$ adapters by $1 / 4$ to reduce the amount of adapter dimer that can form. We found that when not diluted, the $130 \mathrm{bp}$ band intensity increases making it difficult to distinguish from the 150 bp band containing the desired small RNA libraries on a gel.

Another current challenge of preparing sequencing libraries is the accurate quantification of product prior to sequencing. We have found that different methods give varying results on the same library. We suggest that the researchers use multiple methods of quantification to get an accurate estimation of concentration.

This protocol can be widely applied to genetic, developmental studies, or other applications where RNA is being harvested from a low number of cells. This approach simplifies temporal studies by avoiding the pooling of embryos and can easily be applied to both non-sorted and sorted cells.

\section{Disclosures}

The authors have nothing to disclose.

\section{Acknowledgments}

This project was supported by Andrew McDonough B+ Foundation and the NIH (R01-HD099252, R01HD098131. R.J.P. is a CPRIT Scholar in Cancer Research (RR150106) and V Scholar in Cancer Research (V Foundation). The authors would also like to acknowledge the Cytometry and Cell Sorting Core at BCM for providing equipment necessary for this project.

\section{References}

1. Sauka-Spengler, T., Bronner-Fraser, M. A gene regulatory network orchestrates neural crest formation. 
Nature Reviews Molecular Cell Biology. 9, 557-568 (2008).

2. Abrahante, J. E. et al. The Caenorhabditis elegans hunchback-like gene lin-57/hbl-1 controls developmental time and is regulated by microRNAs. Developmental Cell. 4 (5), 625-637 (2003).

3. Lin, S.Y. et al. The C elegans hunchback homolog, hbl-1, controls temporal patterning and is a probable microRNA target. Developmental Cell. 4 (5), 639-650 (2003).

4. Reinhart, B. J. et al. The 21-nucleotide let-7 RNA regulates developmental timing in Caenorhabditis elegans. Nature. 403 (6772), 901 (2000).

5. Slack, F. J. et al. The lin- 41 RBCC gene acts in the C. elegans heterochronic pathway between the let-7 regulatory RNA and the $\mathrm{LIN}-29$ transcription factor. Molecular Cell. 5 (4), 659-669 (2000).

6. Vella, M. C., Choi, E.Y., Lin, S.Y., Reinert, K., Slack, F. J. The C. elegans microRNA let-7 binds to imperfect let-7 complementary sites from the lin-41 3'UTR. Genes \& Development. 18 (2), 132-137 (2004).

7. Ventura, A. et al. Targeted deletion reveals essential and overlapping functions of the miR-1792 family of miRNA clusters. Cell. 132 (5), 875-886 (2008).

8. Eberhart, J. K. et al. MicroRNA Mirn140 modulates Pdgf signaling during palatogenesis. Nature Genetics. 40 (3), 290 (2008)

9. $\mathrm{Li}, \mathrm{Y}$. et al. Dynamic regulation of small RNAome during the early stage of cardiac differentiation from pluripotent embryonic stem cells. Genomics Data. 12, 136-145 (2017).
10. Chugh, P., Dittmer, D. P. Potential pitfalls in microRNA profiling. Wiley Interdisciplinary Reviews: RNA. 3 (5), 601-616 (2012).

11. Werber, M., Wittler, L., Timmermann, B., Grote, P., Herrmann, B. G. The tissue-specific transcriptomic landscape of the mid-gestational mouse embryo. Development. 141 (11), 2325-2330 (2014).

12. Yang, Q. et al. Highly sensitive sequencing reveals dynamic modifications and activities of small RNAs in mouse oocytes and early embryos. Science Advances. 2.6 (2016).

13. Ohnishi, Y. et al. Small RNA class transition from siRNA/piRNA to miRNA during pre-implantation mouse development. Nucleic Acids Research. 38 (15), 5141-5151 (2010).

14. Loontiens, S. et al. Purification of high-quality RNA from a small number of fluorescence activated cell sorted zebrafish cells for RNA sequencing purposes. BMC Genomics. 20 (1), 228 (2019). 